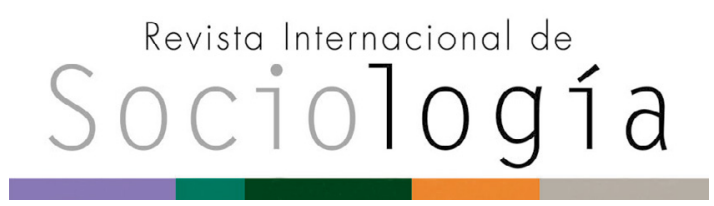

Revista Internacional de Sociología RIS

vol. 79 (2), e182, abril-junio, 2021, ISSN-L:0034-9712 https://doi.org/10.3989/ris.2021.79.2.19.141

\section{LA BIOMEDICALIZACIÓN DE LAS VIDAS DE LAS MUJERES: UNA REVISIÓN DE SUS DIMENSIONES DESDE LAS PUBLICACIONES EN ESPAÑOL}

\author{
Maribel BlÁzquez Rodríguez \\ Universidad Complutense de Madrid (UCM) \\ miblazqu@ucm.es \\ ORCID iD: https://orcid.org/0000-0002-5149-3699
}

\section{THE BIOMEDICALIZATION OF WOMEN'S LIVES: A REVIEW OF ITS DIMENSIONS FROM PUBLICATIONS IN SPANISH}

Cómo citar este artículo / Citation: Blázquez Rodríguez, M. 2021. "La biomedicalización de las vidas de las mujeres: una revisión de sus dimensiones desde las publicaciones en español". Revista Internacional de Sociología 79(2):e182. https://doi. org/10.3989/ris.2021.79.2.19.141

\footnotetext{
Resumen

La biomedicalización es un fenómeno que implica la intervención de los saberes y tecnologías biomédicos en cada vez más aspectos de la vida, con un aumento de su jurisdicción desde la enfermedad a la salud y, hoy en día, sobre la gestión de los cuerpos y las vidas. Con el fin de concretar la complejidad del fenómeno, este artículo apuesta por diseccionar la biomedicalización en diferentes dimensiones: el biologicismo, la patologización, el uso de tratamientos y el control sanitario. El proceso de biomedicalización no ha sido homogéneo, pues no acontece de manera similar ante cualquier condición, cualquier colectivo ni cualquier lugar. Una mirada centrada en las mujeres da cuenta de que este proceso ha afectado a algunas condiciones de sus vidas: menstruación, menopausia, fertilidad, sexualidad, parto, lactancia, cuerpo, salud mental y violencia. Para ello, el artículo aporta algunos trabajos clave del Estado español.
}

\section{Palabras clave}

Medicalización; Biopolítica; Salud de las mujeres; Control médico; Promoción de enfermedades.
Copyright: (C) 2021 CSIC. Este es un artículo de acceso abierto distribuido bajo los términos de la licencia de uso y distribución Creative Commons Reconocimiento 4.0 Internacional (CC BY 4.0).

Recibido: 23/10/2019. Aceptado: 21/07/2020

Publicado: 22/06/2021

\section{Abstract}

Biomedicalization is a phenomenon that involves the intervention of biomedical knowledge and technologies in more and more aspects of life, increasing its jurisdiction from disease to health, and today, in the management of bodies and lives. In order to explain the complexity of this phenomenon, the article focuses on dissecting biomedicalization in different dimensions: biologicism, pathologization, use of treatments and health control. Biomedicalization has not been a homogeneous process, occurring similarly to any condition, any group and any place. An approach focused on women shows how this process affects to some conditions in their lives, such as menstruation, menopause, fertility, sexuality, childbirth, lactation, body, mental health and violence. To this end, the article provides some key works in Spanish State.

\section{KEYWORDS}

Medicalization; Biopolitics; Women's health; Medical control; Disease mongering. 


\section{INTRODUCCIÓN}

En la década de los setenta del siglo XX se inició la denuncia de la medicalización de la vida — siguiendo el concepto propuesto por Ivan Illich (1975) - y la publicación de algunos de los trabajos centrales sobre este tema. Irving Zola (1972) definió la medicalización como el proceso de definir cada vez más aspectos de la vida como salud o enfermedad. Peter Conrad (1975) propone que la medicalización es la definición y el tratamiento médico de comportamientos. Illich (1975) lo asocia al incremento del gasto sanitario, la dependencia de los fármacos y de la asistencia sanitaria. Por último, Michel Foucault (1976) describe que el discurso médico y su práctica están alcanzando cada vez más aspectos del cuerpo y de la vida. Recientemente, y desde la sociología, se han publicado trabajos de revisión del concepto (Bianchi 2019; Busfield 2017; Bell y Figert 2015). En el mismo tiempo, el Movimiento de Salud de las Mujeres planteó, como ámbito central para la liberación de las mujeres, el control sobre sus cuerpos —como refleja el proyecto del Colectivo de las Mujeres de Boston ([1971] 2000)—, y aparecieron dos textos cruciales de Barbara Ehrenreich y Deirdre English ([1973] 1990; [1972] 1981). Desde entonces, con diferentes enfoques, este tema no ha dejado de estar presente en los análisis de los procesos de salud, enfermedad y atención, pero, tal y como Susan Bell y Anne Figert (2010: 107) afirman, la medicalización no se ha dado de la misma forma en los diferentes contextos ni respecto a las diferentes poblaciones. Mostrar algunas de estas particularidades, refiriéndonos a las mujeres como un colectivo afectado de modo específico por la medicalización, será uno de los propósitos de este artículo, como hacen las sociólogas Catherine Riessman (1983) y Elianne Riska (2010; 2003). Esta última indica que ha habido tres fases en la incorporación de una mirada de género en la medicalización: la primera, centrada en que la medicalización se consideraba neutral; la segunda visibilizó el androcentrismo de la medicina, mientras que la tercera, a partir de la década de 1990, reconoció la participación de las mujeres en estos procesos y la medicalización de los hombres. La antropóloga Margaret Lock (2001: 481) señala que el feminismo llamó a las ciencias sociales a contribuir a despertar la conciencia acerca de lo inapropiado de este proceso y, por tanto, a su resistencia.

Ellen Annandale y Anne Hammarström (2015) proponen que el estudio de la biomedicalización se haga desde una mirada específica de género que tenga en cuenta las diferencias entre los hombres y las mujeres no solo en las enfermedades, sino también en los riesgos y en los tratamientos - como los farmacológicos-. En nuestro contexto se habla de los sesgos de género (García Dauder y Pérez Sedeño 2017; Ruiz-Cantero y Verbrugge 1997), que han producido una paradoja: las mujeres están más sometidas a controles sanitarios, particularmente en lo reproductivo, pero como las diferencias no han sido estudiadas, están peor atendidas. Carme Valls (2010: 160) habla de la morbilidad diferencial, es decir, el conjunto de enfermedades, motivos de consulta o factores de riesgo que merecen una atención específica hacia las mujeres, bien porque solo ellas pueden presentar dichos problemas (embarazo, parto, menstruación), bien porque sean mucho más frecuentes, como lo son, por ejemplo, las anemias, el dolor crónico, las enfermedades autoinmunes, las endocrinológicas, la ansiedad y la depresión.

A menudo se aborda la medicalización como si fuera un embrollo (Sholl 2017), caracterizado por la (omni)presencia tanto de la medicina - siendo un saber, una institución y una profesión- como de sus acciones - diagnóstico y etiquetaje de enfermedades, tratamientos (curativos y preventivos)-. En este artículo se pretende ordenar este enredo al poner el foco en las dimensiones que se han desarrollado - y que se continúan desarrollando-, haciendo que la biomedicalización disponga de una relación específica con las mujeres.

Adopto el término biomedicalización siguiendo a Adele Clarke y su equipo (2010; 2003), quienes subrayan que este proceso se da a partir de la década de 1980 en EE.UU con el desarrollo de complejos tecnocientíficos en los que la biología y la tecnología, con sus diferentes ramas, adquieren un papel central. También conlleva un enfoque de salud, riesgo y vigilancia. Estos hechos producen una transformación tanto del conocimiento médico como de sus intervenciones, trayendo nuevos criterios de poder y autoridad que, a su vez, originan transformaciones en los cuerpos e identidades que se conectan con el biopoder y las biopolíticas foucaultianas para regular las vidas.

\section{Dimensionando la biomedicalización}

La socióloga Jennifer Torres (2014: 161) distingue cuatro dimensiones de la medicalización, las cuales están articuladas en la realidad y no son autónomas unas de otras, a saber: la descripción en términos médicos de cualquier situación o condición, la patologización, el tratamiento y el control por parte de los profesionales o instituciones. Esta distinción no solo permite entender mejor cómo se produce la biomedicalización, sino advertir que habría diferentes niveles o estrategias y, a su vez, procesos de desmedicalización (Bell y Figert 2015; Armstrong 1995; Fox 1977). De modo que la biomedicalización, en algunos casos, puede ser parcial; hecho que resulta crucial en esta mirada centrada en las mujeres, pues arroja luz no solo acerca de cómo ellas han participado en la medicalización de sus vidas como estrategia para mejorarla, sino también de cómo han buscado, y llevado a cabo, formas de contestación. El sociólogo Drew Halfmann (2012) revisa cómo las acciones 
de medicalización y desmedicalización del aborto en EE.UU se han dado simultáneamente, pero las primeras han sido más visibles y estudiadas, como el control jurídico y médico del mismo, frente a las segundas, como la disminución del control apoyado en los derechos de las mujeres. Asumiendo esta interacción entre ambos procesos, me centraré en las dimensiones que conformarían la biomedicalización, siguiendo el planteamiento de Torres.

\section{Primera dimensión: la supremacía de la bio- logía en la definición de las condiciones de vida de las mujeres}

La definición de diferentes condiciones en términos biológicos ha sido una de las formas básicas de biomedicalización. El término 'condición' es el que se emplea en antropología en sustitución de enfermedad, problema o salud y permite analizar cómo ciertas situaciones del ciclo vital de las mujeres han sido medicalizadas (Montes 1999), como se verá en una primera parte (menstruación, embarazo, sexualidad, menopausia, fertilidad y cuerpo). Posteriormente se plantea que determinadas perspectivas sobre estas cuestiones conducen a una visión esencialista y reproductiva de las mujeres. Esta elección conduce a excluir otras condiciones que afectan tanto a hombres como a mujeres y sobre las que no habría una mirada específica de género.

\section{La menstruación}

Ha sido descrita de una forma negativa. Emily Martin (1987) explica que se piensa como un proceso fallido desde un punto de vista reproductivo. En las definiciones médicas se concibe básicamente como un proceso fisiológico mediado por hormonas, en el que no se considera que estas puedan estar afectadas por diferentes factores como la exposición a contaminantes (Valls 2018: 394-398), el estrés (Valls 2010: 134) u otros. Tampoco se incluyen las concepciones culturales acerca de la menstruación, que en algunos casos se alejan de la biología; así, Els Bransen (1992) entrevistó en los Países Bajos a 21 mujeres para conocer la importancia del ciclo en sus vidas, encontrando que describían la menstruación en sus propios términos, es decir, como un proceso natural que no solo tiene que ver con las hormonas, distanciándose así de los saberes médicos expertos. Miren Guilló (2014), que ha llevado a cabo una investigación sobre políticas alternativas de la menstruación, recoge y analiza concepciones de algunas jóvenes que se alejan de la visión patológica y sucia de la sangre menstrual. Otros estudios muestran que las concepciones negativas y patológicas de la menstruación influyen en la posible decisión de suspender el ciclo menstrual (Martínez, Parera y Rius 2018; Oinas 1998).

\section{El embarazo, parto y lactancia}

Aunque se postule que estos procesos no son enfermedades sino acontecimientos "normales" del cuerpo de las mujeres, esta visión colisiona con lecturas del embarazo que concentran las explicaciones en términos orgánicos que deben ser vigilados para detectar la aparición de riesgos, que lo someten a la realización de pruebas, a un seguimiento médico aunque sea bajo la mirada de las matronas, junto a la ginecología- $\mathrm{o}$ al control sanitario en los hospitales y con tecnología biomédica. Estas acciones manifiestan que se entiende el embarazo más como un proceso médico que sociocultural (Blázquez 2010; 2005). Torres (2014: 162) expone que la lactancia materna también se describe como un proceso que tiene que ver con unos órganos bajo una función hormonal, con un valor nutricional y unos efectos beneficiosos en relación con la salud y la enfermedad.

\section{La sexualidad}

Tampoco ha escapado de esta mirada médica. Desde los trabajos de Kinsey y Masters y Johnson, determinantes en la visibilización de esta dimensión del cuerpo o de la vida, es entendida más como un proceso neurofisiológico (Zuluaga, Cruz y Meneses 2018: 63) al que habría que incorporar miradas socioculturales (García Dauder y Pérez Sedeño 2017; Freixas y Luque 2009). Otro ejemplo en nuestro país podría ser el cambio en la conceptualización derivada de la aprobación de la Ley Orgánica 2/2010, de 3 de marzo, de salud sexual y reproductiva, en la que se pasa de hablar de derechos sexuales y reproductivos a salud sexual y reproductiva. Esta ley es un marco fundamental para la defensa de estos derechos, pero la ubicación de la sexualidad dentro de la salud legitima tanto su conceptualización biológica como sus intervenciones sanitarias.

\section{La menopausia}

También queda dominada por la biología y alejada de las individualidades y de los contextos. Lock (1993) plantea en su estudio comparativo entre las mujeres japonesas y estadounidenses que lo único común entre ellas es la disminución de los estrógenos y la supresión de la menstruación; el resto de las variaciones, incluso sus manifestaciones, se deben a múltiples factores como los significados culturales que se han atribuido a esta etapa y las estrategias de biomedicalización de cada contexto. Otros trabajos cuestionan la biomedicalización de la misma (Freixas 2007; Delgado et al. 2001; Esteban 2001; Rueda 1997).

\section{La fertilidad}

Se plantea como un problema por cuanto la maternidad aún es considerada un eje central en las vidas de las mujeres, cuestión largamente tratada y denunciada por la teoría feminista. Además, se atri- 
buyen a ellas las causas de los problemas de fertilidad, por una parte, porque la virilidad no puede ser cuestionada, convirtiéndose así la infertilidad en un tabú social, y, por otra, porque ha sido más estudiada en relación con las mujeres (Bell 2016).

\section{El cuerpo}

El cuerpo de las mujeres ha sido definido desde un determinado modelo de belleza y perfección, lo que las conduce a que busquen tratamientos de corrección o mejora (Hogle 2005) —aunque los hombres cada vez estén más incluidos en estos mandatos (Coll-Planas, Alfama y Cruells 2013; Liévano 2012; Muñiz 2010) - Se ha construido la idea de que los cuerpos no delgados constituyen un problema, y la grasa es un problema situado en las mujeres (Wolf 1991: 192), por lo que son consideradas menos femeninas, menos bellas, y menos deseables. La gordura se valora como fuente de insatisfacción y, así, cada vez más niñas a edades más tempranas empiezan a regular su peso y a seguir dietas (Zafra 2015). La antropóloga Mónica Ramos (2017: 172176) señala que estas visiones suponen un doble rasero para las mujeres mayores, pues quedan fuera de la asociación juventud-salud-belleza.

El tamaño de los senos también se problematiza bajo una mirada médica que justifica su relación con la baja autoestima y las alteraciones psicosociales. Otro tema silenciado es el tamaño de los labios menores, que el ginecólogo Jorge López-Olmos (2013) indica que se presenta en chicas adolescentes cada vez más jóvenes, de entre 10 y 12 años, fundamentado en molestias. Este autor se interroga si se debe más a cumplir criterios de aceptación social derivados de la industria pornográfica y las modas que a problemas funcionales. Así, el recorte de los labios menores se ha convertido en una cirugía común en EE.UU

Distingo esta primera dimensión de la biomedicalización de otras como la patologización o el uso de los tratamientos porque, como dan cuenta estos ejemplos, implica entender la vida principalmente en términos biológicos. Didier Fassin (2011) refuerza esta idea al indicar que la medicina problematiza de una determinada manera la realidad; por ejemplo, la psicóloga Linda Gannon (1998: 295) señala que el embarazo se conceptualiza como un proceso saludable, mientras que la menopausia se presenta como insalubre o como una enfermedad, perpetuándose así el mandato de que las mujeres sean reproductoras, pues en ello está también en juego su salud. A este respecto, Esteban (2006: 11) habla de la naturalización social de las mujeres, para subrayar la "percepción absolutamente esencialista y biologicista del cuerpo humano". Marcia Inhorn (2006: 350) se refiere a ella como la esencialización de las vidas de las mujeres, pues estas son definidas con base en esta característica —la posibilidad de embarazar- se, dar a luz y reproducirse-, sin tener en cuenta que hoy en día no todas las mujeres serán madres; ni de que, de las que lo serán, no todas tendrán un embarazo, ni un parto, ni quizá darán de lactar.

Belén Nogueiras (2018) señala que pensadoras feministas - desde Mary Wollstonecraft a, en nuestro país, Inés Joyes (Madrid, 1731-1808) — han denunciado la atribución de la subordinación de las mujeres a una biología diferencial y reproductiva; la naturalización o esencialización ha sido uno de los principales mecanismos empleados a lo largo de la historia y que persiste en nuestros días. Afortunadamente, aparecen propuestas — como la de la médica Sara Velasco (2009) - que insisten en entender las vidas de las mujeres y, principalmente, sus malestares, dentro de teorías biopsicosociales y de género que incluyen el contexto social y la subjetividad. Lock (1993), por su parte, propuso hablar de biologías locales, refiriéndose a que los procesos biológicos son el resultado de interacciones con los ambientes en los que las personas han vivido, que se enredan inseparablemente con el tiempo. Por tanto, el desafío sería "apoyarse en lecturas de las propias mujeres y hombres que reflejaran maneras de entender y vivir los padecimientos mucho más diversas y ricas en matices y factores condicionantes (clase social, etnia, edad, experiencia laboral, migración, formas de convivencia, sexualidad...)" (Esteban 2006: 13).

No obstante, hoy estamos ante la construcción de un sí mismo biológico (Rodríguez 2017: 91) particularmente determinado por subcampos como la genética, la inmunología y las neurociencias. Así, la genetización, como refiere la epidemióloga Abby Lippman (1991), se ha convertido en un proceso continuo por el cual las diferencias biológicas y socioculturales entre individuos se reducen a sus códigos de ADN y las tecnologías genéticas se adoptan para dar respuestas ante la vida. Lucien Sfez (2008: 195) sugiere que esta es una de las utopías de la modernidad y nos conduce a la búsqueda de la salud perfecta. En el caso de las mujeres, está muy presente en aquellas que quieren ser madres, para revisar si son portadoras de determinadas enfermedades y, en caso afirmativo, optar por determinadas intervenciones en la reproducción y en los test de screening del embarazo. La genetización también se percibe en el cáncer de mama, en que cada vez se da mayor relevancia al cribado de los genes (Gibbon 2006). Paula Sibilia (2016: 104) indica que esta concepción de la causalidad inscrita en la programación genética -o en los flujos cerebralesconduce al abandono de una visión holística y a una mirada del cuerpo micromolecular presente en la investigación con células madre, los bancos de sangre de cordón umbilical, la reproducción asistida y la congelación de óvulos. Se trata de bioeconomías (Waldby y Cooper 2008), ya que convierten los cuerpos en bienes dirigidos a la prevención, regeneración y curación, afectando más a los cuerpos reproductivos. 
Esto conduce a que se hable de healthización (Crawford 1980), saludización (Williams 2001; Armstrong 1995) o sanitarización (Blázquez y Cornejo 2014; Camargo 2013; Fassin 2011), en el sentido de que en toda la experiencia humana prima una definición orgánica —alimentación, ejercicio físico, sueño, sexualidad, entre otros-, de modo que se sanitariza sin patologizar, es decir, se describe en términos biológicos pero no de enfermedad (Rose 2007), y estaríamos ante el imperativo de la salud, como sugirió Deborah Lupton (1995). Flavia Costa (2017: 125) entiende que el vínculo con el cuerpo dispone de un triple objetivo: mejorar e intensificar la vida, estetizar el cuerpo y aumentar su potencial en cuanto capital humano. Se trata de ejercer un control del cuerpo a través de la alimentación, el ejercicio físico y cualquier tipo de autocuidado. La difícil delimitación entre lo que es salud y lo que es enfermedad es debatida por Jenny Hislop y Sara Arber (2003) al interrogarse sobre si el abordaje del sueño en las mujeres es medicalización (pues produce enfermedades y problemas) o salubrización (pues es una condición para la salud); para las autoras, la diferencia radica en que el segundo enfoque está centrado en las decisiones de los sujetos y las estrategias que adoptan para manejar las condiciones sociales que inciden en el sueño.

Definir en términos biológicos cualquier condición puede ser ya una forma de biomedicalización, incluso sin el desarrollo de las otras dimensiones, pero sí es necesaria para que se puedan dar las otras.

\section{Segunda dimensión: la patologización o la definición de una condición en términos de enfermedad}

El biologicismo crea las bases para etiquetar aspectos de la vida como indicadores de salud o enfermedad, como signos y síntomas, de males a enfermedades, de la ley a la medicina, siguiendo la advertencia que ya realizó Zola (1972). Patologizar cualquier proceso supone establecer una normalidad, y aquello que se salga de ahí se define como anormal. Esto es lo que ha sucedido, por ejemplo, con la menstruación: sin estudiarse ni haberse establecido cuál es el patrón normal de la menstruación, salvo el que plantea la médica Valls (2010: 135-136) basado en mujeres suecas y danesas, se etiquetan como anormales el dolor, las molestias, la irregularidad y el sangrado abundante, sin ser atendidos a pesar de las quejas de las mujeres (Blázquez y Bolaños 2017). O se establecen enfermedades como el síndrome poliquístico o premenstrual (García Porta 2006; Caplan, McCurdy-Myers y Gans 1992).

Eliot Freidson ([1970] 1978: 254) ya había planteado que la medicina era activa en la producción de enfermedades y no solo esperaba a que estas fueran presentadas por los pacientes, sino que la ambición de los médicos era buscar y descubrir una enfermedad con la que inmortalizar su nombre.

En el ámbito de la medicina, los conceptos de medicalización y patologización se han visto desplazados por otros como el de disease mongering o promoción / invención de enfermedades, acuñado por la periodista Lynn Payer. Posteriormente, el periodista Ray Moynihan lo adoptó y publicó en las principales revistas médicas, retomando y ampliando las diferentes estrategias de la promoción de las enfermedades. Estas estrategias son cuatro: la primera, la ya mencionada tendencia en la que distintas condiciones son clasificadas como problemas biológicos; la segunda, el establecimiento de la anormalidad y sus síntomas; la tercera, la definición de riesgos y, por último, la cuantificación de los problemas.

\section{El establecimiento de la anormalidad y sus sín- tomas}

Cuando una condición se define como anormal y, por tanto, como patología, hay que establecer cuáles son los síntomas de la desviación de la normalidad. Moynihan, Heath y Henry (2002) señalan que los síntomas se sobredimensionan o se exagera su gravedad, con ejemplos como el síndrome del intestino irritable, la ansiedad o la disfunción eréctil. La médica Teresa Ruiz-Cantero et al. (2019) subrayan que el síndrome de intestino irritable es una enfermedad que requiere de una mayor investigación incorporando los sesgos de género, pues la prevalencia es dos veces mayor en las mujeres respecto a los varones. En consecuencia, apuntan, habría que incluir muestras con representaciones de ambos sexos y hacer un buen diagnóstico diferencial que mostrase si realmente afecta más a las mujeres, además del cómo y el porqué. Respecto a la ansiedad, Valls (2010: 295-297) plantea el género como un factor de riesgo fundamental para la presentación de sintomatología depresiva con una ratio 2:1 mujer/varón, y sugiere revisar algunos parámetros biológicos (como la anemia o la tiroides) junto a los roles de género como la doble jornada, el trabajo doméstico o el de cuidadoras que intervienen en su aparición.

\section{La definición de riesgos}

La tercera de las estrategias de promoción de las enfermedades tiene que ver con la definición de riesgos como enfermedades, aunque estos no lo sean y constituyan solo probabilidades. Uno de los casos más citado es el de la osteoporosis. El médico Roberto Sánchez (2012: 619-621) afirma que el riesgo de fractura en mujeres menopáusicas es mínimo, aunque hayan tenido ya una fractura previa. Este autor apunta que, en lugar de desarrollar estrategias de prevención de caídas, lo que se hace es realizar pruebas como las densitometrías a las mujeres perimenopáusicas y, a partir de un valor establecido, 
se les pone en tratamiento. Esto le lleva a hablar de los siete mandamientos de la "miedicalización", coincidiendo con otras propuestas que hablan de la creación del pánico estadístico en cuanto al uso de los números y los datos estadísticos para reforzar la experiencia de riesgo (Woodward 1999), que es la siguiente estrategia.

\section{La cuantificación del problema}

Otra forma de promover enfermedades es presentar el número de personas a quienes afecta ese riesgo, síntoma o condición patologizada, para generar preocupación en la población sana. Un ejemplo es la disfunción sexual femenina, que, de acuerdo a un estudio en EE.UU afectaba al 43 \% de la población femenina en 1999 (Laumann, Paik y Rosen 1999). Leonore Tiefer (2006) indica que, desde la década de 1970, la medicina empieza a publicar diferentes trabajos sobre las deficiencias en la sexualidad de las mujeres, para posteriormente difundir la existencia de productos médicos que los resuelven, como también muestran otros trabajos (Miglioranza 2018; González García 2015). Otros estudios (Zuluaga, Cruz y Meneses 2018: 55-64; Riska 2010: 158) señalan que esta estrategia también se ha desarrollado en la andropausia y en la disfunción sexual masculina, aunque con menor éxito, ya que no iban acompañados de tratamientos médicos, lo que conformaría la siguiente dimensión.

\section{Tercera dimensión: el uso de tratamientos e intervenciones biomédicas}

Consiste en el uso de tratamientos o intervenciones biomédicas basadas en fármacos, técnicas y tecnologías.

\section{La farmacologización}

Es entendida como un proceso social ligado al de la biomedicalización, pero con su propia entidad, ya que depende de la creación de unas circunstancias tanto culturales como sociopolíticas que permitan su uso. La cuestión ha sido ampliamente analizada por diferentes autores (Bianchi 2018; Camargo y Reid 2016). La socióloga Tasleem Padamsee (2011) habla del buen trabajo de las corporaciones farmacéuticas en la transmisión de mensajes dirigidos a las mujeres para que controlen mediante fármacos procesos como la fertilidad, la infertilidad y la menopausia, a los que hay que añadir la menstruación. Mención especial requiere la mayor prescripción de psicofármacos a las mujeres (Romo y Gil 2008). Ya el psiquiatra Thomas Szasz ([1970] 1974) había denunciado que diferentes problemas de la vida se trataban como enfermedades mentales. Psicólogas y psiquiatras han defendido que la salud mental de las mujeres no está en relación con una biología diferencial, sino con su discriminación y sus peores condiciones de vida (Pu- jal, Albertín y Mora 2015; Burin 1990; Sáez 1988). No obstante, se interviene poco sobre estas causas y los fármacos se convierten en la principal respuesta sanitaria. En ello, como subraya Margarita López (s.f.: 4), coordinadora de la Red Caps de Mujeres Profesionales de la Salud, influye que no se hace apenas "investigación no farmacológica, a pesar de que la poca que hay (intervenciones psicológicas, sociocomunitarias...) demuestra con frecuencia ventajas curativas, así como económicas, y con muchos menos efectos secundarios indeseados".

En ese sentido, es oportuna la propuesta que desarrolla Jessica Shipman (2010: 1331) siguiendo el planteamiento de Nikolas Rose (2007: 700) de huir de marcos dualistas que entiendan estos tratamientos como buenos o malos, beneficiosos o perjudiciales. En su estudio sobre mujeres que decidieron seguir tratamientos de supresión de la menstruación, Shipman apunta que no hay que valorar la agencia de las mismas solo como resistencia o desafío: también implica que el uso de estos fármacos puede ser útil en sus vidas. Las transformaciones, en su opinión, no vienen del cuestionamiento de esta dimensión de la farmacologización, sino de la contestación del significado, donde rompen con la mirada biomédica que entiende la menstruación como un proceso reproductivo y patológico; es decir, en relación con la primera y segunda dimensión planteadas, no con la tercera. De modo que se pueden observar relaciones y contradicciones entre las diferentes dimensiones.

\section{El intervencionismo en el parto}

El caso más abordado respecto a la incorporación de la intervención médica en la vida de las mujeres ha sido el parto. Para entender cómo se produce, hay que tener en cuenta el proceso previo de control de los saberes de las sanadoras, quienes históricamente han atendido los partos. A partir del siglo XII se crean en Europa las universidades vinculadas a la Iglesia. La medicina comienza su institucionalización y profesionalización, lo que conlleva la prohibición y persecución de estas practicantes no tituladas, que eran principalmente mujeres y pobres. Ehrenreich y English ([1972] 1981: 25) describen que una de las acusaciones de la caza de brujas era que tenían saberes mágicos y podían curar. En este proceso, los profesionales hombres, procedentes de familias acaudaladas, presionaron contra la posible competencia de las mujeres y recibieron el apoyo de los estamentos privilegiados (la Iglesia y el Estado). En EE.UU fue crucial el Movimiento Popular de la Salud del siglo XIX donde estaban mujeres, hombres de clase baja y negros, quienes sin titulación ejercieron hasta el siglo XIX. El desplazamiento de las mujeres y otros practicantes siguió diferentes estrategias, desde la prohibición y persecución hasta el ofrecimiento de otros tratamientos por parte de los nuevos médicos titulados (fórceps, anestesia, cesárea). Riessman (1983) señala que las mujeres 
querían librarse no solo del dolor, sino de la posibilidad de la muerte, en un contexto de baja natalidad de las clases altas y elevada mortalidad materna y fetal -lo que suponía una enorme preocupación en los siglos XVIII y XIX- También se buscaba que el parto fuera más fácil, siendo la clase médica la que ofreció estas seguridades, y no las parteras tradicionales. Judith Leavitt (1980: 153-154) plantea que esta es la ironía, que el control por parte de estos profesionales sirviera para distanciar a las mujeres de sus propios cuerpos. Estos trabajos muestran que algunas de las mujeres que lideraron la incorporación de la clase médica a los partos fueron las sufragistas. Paradójicamente, en los años setenta del siglo pasado, y en otro contexto diferente, otras mujeres, también de la lucha feminista, reclamaron el retorno de los partos a los domicilios, su destecnologización y la participación de las matronas (Colectivo de Boston [1971] 2000).

En España, Teresa Ortiz (1992: 324), historiadora de la medicina, sitúa la entrada de los cirujanos en los partos en el siglo XVIII; primero en la nobleza, luego en las ciudades y posteriormente en todos los partos, con la prohibición de la actuación de las matronas de forma independiente. Esta prohibición se intensificó a partir de la creación de la Cátedra de Partos en el Real Colegio de Cirugía de San Carlos en 1780 para la formación de las matronas bajo el control de los médicos (Ruiz y Gomis 2017). Además, Mary Nash (2000) y Carmen Colmenares (2009) han estudiado cómo el discurso médico se dirigió a las mujeres, culpabilizándolas de la enfermedad y mortalidad infantiles por su falta de conocimientos sobre la crianza. Los saberes empíricos tradicionales sobre el embarazo, el parto y los cuidados infantiles debían ser ahora sustituidos por el saber científico y patriarcal que la nueva medicina e higiene traía. También se transmitieron, durante el franquismo, enseñanzas de higiene, puericultura y maternología, tanto en las escuelas como en conferencias radiadas y charlas. Se procuró que los cuidados fueran no solo tareas asignadas a las mujeres, sino que estas interiorizaran la necesidad del saber médico, puesto que los saberes para enfrentar el embarazo y la crianza eran técnicos. A partir de ahí, las mujeres -y gran parte de la ciudadanía - fueron colocadas en una situación de incapacidad para sus cuidados (Ortiz 2006: 69-72; Esteban 2001: 35; Juan 1991: 44). La desaparición del parto domiciliario se sitúa también en relación con el Seguro Obligatorio de Maternidad de 1923 (Ruiz y Gomis 2017: 59) con el inicio de los controles por parte de los médicos en el embarazo, a lo que siguieron las políticas pronatalistas en la primera etapa del franquismo, en las que jugó un papel decisivo la afiliación al Seguro Obligatorio de Enfermedad desde finales de la década de 1940 (Ruiz y Gomis 2017). Así, Juan Bosch Marín et al. (1950, citado en Barceló y Montes 2016: 12) estimaban que, de 1947 a 1949, el 94,1 \% de los partos fueron aten- didos en el domicilio, mientras que en residencias hospitalarias solo se atendió el 5,1\%. No obstante, la cobertura de ocho días de ingreso gratuito para partos normales, la creación de nuevas y modernas clínicas del Seguro y la construcción de nuevas residencias maternales favorecieron la creación de una cultura a favor del control médico, donde también participaron las propias matronas, que aceptaron la supeditación a los médicos. De este modo, en 1969 ya el $44 \%$ de los nacimientos se producía en los hospitales (De Miguel 1980: 39); en 1985, el porcentaje asciende al 82,5\%, y en 1995 ya correspondía al 99,1 \% (Llácer 2000: 1).

En España, a diferencia de otros países como EE.UU. (Riessman 1983; Ehrenreich y English [1972] 1981; Leavitt 1980), el control médico de los partos no fue a través de la anestesia epidural. Esta fue poco utilizada en los partos hospitalarios (González, Fernández y Gómez 2015) hasta que en 1998 el ministro de Sanidad anunció su disponibilidad en todos los nacimientos a partir del año 2000 , de tal forma que una política pública de igualdad en salud era garantizar el acceso a todas las mujeres que lo solicitaran dentro de la red pública. Apenas unos años después, en la Estrategia de Atención al Parto Normal del Ministerio de Sanidad y Consumo (2007), se pronuncia, como una política también de igualdad en salud, la disminución del uso de la anestesia epidural en los partos. En la misma participaron asociaciones de mujeres, profesionales y expertos bajo la dirección del Observatorio de Salud de la Mujer, con la finalidad de diseñar una política para disminuir la medicalización del parto y promover una conceptualización fisiológica del parto, desde un enfoque de género y diversidad.

Por tanto, los objetivos de una agenda feminista en la salud para el control de sus cuerpos se concretan en función de diferentes aspectos: según los contextos históricos, los países, las características de las propias mujeres (nivel de ingresos, de estudios, edad y ocupación) y los significados ante una misma intervención médica, como la anestesia epidural (Biedma, García y Serrano 2010: 6).

\section{El intervencionismo médico en la lactancia}

La lactancia materna también está afectada por esta dimensión al incorporarse toda una serie de tecnologías (extractores de leche, protectores de pezón, jeringas de alimentación y productos farmacéuticos que estimulan la producción), aunque se considere como un proceso fisiológico y su gestión sea con leche materna (Torres 2014). Carmen Romero y Pablo Santoro (2018), en su estudio de las diferentes tecnologías que se incorporan en la gestión de la lactancia, ponen el foco en los bancos de leche materna. En este análisis indican que la leche se entiende como un alimento, un fluido, un tejido corporal y un 
medicamento, y, en función de su definición, estaríamos más próximos a la desmedicalización.

\section{El intervencionismo en la alimentación}

Paula Rodríguez Zoya (2017) habla del concepto de medicamentalización alimentaria como fenómeno que articula la medicalización de los alimentos y de la alimentación. El primero concibe a los alimentos desde sus propiedades nutricionales, es decir, desde la biología y como medios para la optimización de la vida (primera dimensión). El segundo conlleva la patologización de diversas condiciones (segunda dimensión) ante los que se plantea intervenir (tercera dimensión) a través de la regulación no solo de qué se come, sino de cómo se come (Gracia 2007).

\section{El intervencionismo médico a través de los con- troles sanitarios}

Sánchez (2012) desarrolla varios ejemplos referidos tanto a tratamientos médicos como a los controles sanitarios que una mujer no necesita, entre los que se encuentran los cribados de cáncer de mama y de cérvix, el tratamiento de la osteoporosis, la vacuna del VPH y la terapia hormonal sustitutiva (THS). Respecto a este último tratamiento, la médica Carmen Mosquera (2009) indica que antes del comunicado de la Agencia Española del Medicamento en el 2004, que recogía los resultados de las investigaciones científicas que alertaban de las enfermedades que generaba este tratamiento (cáncer de mama, cáncer de endometrio, infarto de miocardio, enfermedad vascular cerebral y tromboembolia venosa), se estimaba que un $17 \%$ de las mujeres asturianas usaban estos compuestos hormonales. Posteriormente, con la iniciativa de difundir este comunicado tanto entre profesionales como mujeres, se observó un descenso del 73,6\%. Cabría preguntarse acerca de si este descenso tan alto era debido a que no todas las mujeres a quienes les prescribieron este fármaco lo necesitaban.

\section{Cuarta dimensión: el control sanitario de los cuerpos de las mujeres}

Como consecuencia de las anteriores dimensiones, ciertas condiciones estarían bajo la vigilancia, atención e intervención de los profesionales sanitarios. Por un lado, esta dimensión sitúa la legitimidad de la mirada biomédica, sustentada en conceptualizar el cuerpo desde la biología, establecer una anormalidad, unos riesgos que deben ser visualizados mediante tecnologías, junto a los tratamientos para las patologías reales o posibles. Por otro lado, reclama la participación sanitaria para manejar y gestionar la vida, es decir, para mejorar las condiciones normales: "La personalidad misma se vuelve cada vez más somática" y "vivir bien hoy es vivir a la luz de la biomedicina" (Rose 2013: 7). Ya Deborah Find- lay (1992: 121) sugería que, al ampliar la dominación médica de la enfermedad a la normalidad (primera dimensión) y al establecimiento de riesgos (segunda dimensión), se aumentó la autoridad médica de los cuerpos a las psiques, las familias, los hogares, las redes sociales y al trabajo. Las propias mujeres son las que monitorean sus cuerpos y, después de ello, depositan sus cuerpos en los profesionales de la medicina y disciplinas afines, pues consideran que son quienes poseen el verdadero poder y las técnicas de vigilancia sobre los mismos (Lock 2004: 120).

\section{La contextualización de los procesos de control}

Clarke et al. (2010) señalan que los cambios en la biopolítica planteada por Foucault se relacionan con dos hechos: uno, el Estado ya no es el único agente en el control de la normalidad de las poblaciones; y dos, las industrias farmacéuticas, las aseguradoras, la academia, los grupos de defensa de los derechos de pacientes, los medios masivos de comunicación, los complejos médico-industriales y sus capitales de inversión son quienes "ahora se disputan el campo de la definición de la salud y la enfermedad y la organización de los cuidados" (Murguía, Ordorika y Guerrero 2016: 105). No obstante, quisiera cuestionar la homogeneidad de este proceso en cualquier sociedad; en España, el Estado se fue haciendo cargo de la salud poco a poco a lo largo del siglo pasado, pero la creación de un sistema de salud universal, público y estatal no acontece hasta el año 1977. Posteriormente se desarrolló la Atención Primaria, las transferencias a las Comunidades Autónomas, la coordinación sanitaria y, como apunta el médico Javier Segura (2018), hasta el año 1995 no llegó el proceso de liberalismo sanitario, es decir, la participación de entidades privadas en la atención a la salud. Además, conviene subrayar que este proceso ha sido desigual y diferente según los territorios, iniciándose en las comunidades de Madrid y Valencia. Por tanto, no conviene hacer extrapolaciones acerca del desarrollo de las biopolíticas de otros contextos al nuestro. Y, en nuestro país, el movimiento de consumidores surge a finales de la década de 1970 (Llovet 1991) sin la relevancia que ha tenido en otros países, como EE.UU, respecto a la salud, por ejemplo, en la denuncia de la seguridad y los impuestos de los productos sanitarios de la menstruación (Felitti 2016; Bobel 2009).

En España, el control de los partos y otros procesos de las mujeres no solo ha estado bajo el gobierno de los médicos — pues hasta fecha reciente eran solo varones y, hoy en día, el androcentrismo es central en la ginecología y obstetricia, aunque haya mayor presencia de mujeres-, sino también de la Iglesia, quien, al servicio del Estado y junto al propio Estado - con las políticas pronatalistas franquistas - no han permitido a las mujeres pensar -ni elegir-sobre la maternidad y sus cuerpos. Además, a diferencia de otros países, la legalización de los 
anticonceptivos no llega hasta 1978 (Ortiz e Ignaciuk 2018) y el derecho a la interrupción voluntaria del embarazo no lo hace hasta 2010 (Nogueiras 2018), hechos que han marcado la relación de las mujeres con sus cuerpos y su autonomía.

\section{Los efectos del control sanitario}

La antropóloga Inhorn (2006: 356) afirma que toda esta gestión médica, que se da también en otros contextos, realmente no está mejorando las vidas de las mujeres, sino que más bien lo que está produciendo es una vigilancia continua sobre sus vidas. Por consiguiente, corresponde plantearse, como hizo Illich (1975), la iatrogenia que produce la biomedicalización, teniendo en cuenta que se dan efectos positivos y negativos, derivados tanto del buen hacer como de la falta de conocimientos o práctica, tanto de los efectos actuales como de los futuros previsibles e imprevisibles, pero que sugieren que indudablemente el control biomédico no es neutral ni inocuo. Algunos efectos son conocidos y deberían ser informados a las mujeres para tomar decisiones, como plantea López (2002) respecto a las donaciones de óvulos (síndrome de hiperestimulación ovárica, infertilidad, menopausia prematura o cáncer de mama y ovario). Otro ejemplo serían las campañas del cáncer de mama que, como apunta Ana Porroche (2017), transmiten una información sesgada, confusa y estereotipada.

Otros efectos no son tan tangibles, como indica la socióloga Paige Sweet (2014) respecto a la violencia de pareja contra las mujeres. En nuestro país, se realizó un protocolo sanitario con una participación interdisciplinar que empezó a reconocer la violencia, a detectar los casos de mujeres maltratadas, a acompañarlas y a tratar sus problemas desde el ámbito sanitario, ya que previamente no había ni reconocimiento del problema ni atención, aunque se habían desarrollado casas de acogida y una asistencia no institucionalizada (Nogueiras 2018: 248). Sweet se interroga acerca de las consecuencias de introducir esta condición bajo el control sanitario. El hecho de que estas mujeres hayan quedado registradas y su historia clínica siempre muestre este episodio hace que sea un marcador eterno que las sujeta tanto a una vigilancia biomédica no deseada como a una asociación constante de sus vivencias con esta experiencia, es decir, todas sus dolencias actuales y futuras quedarán vinculadas con el maltrato recibido, incluso cuando hayan dejado la relación o haya pasado tiempo. De este modo, debemos prestar atención a los efectos a medio y largo plazo de condiciones como la violencia -o los derechos sexuales que he planteado anteriormente-que, por diferentes razones, han sido biomedicalizadas.

De las cuatro dimensiones de la biomedicalización abordadas, considero que las dos primeras -la definición de las condiciones de vida de las mujeres en términos biológicos y la patologización de estas condiciones con la construcción de riesgos o desviaciones de la normalidad - conducen a las otras dos -al uso de tratamientos y tecnologías médicas y al control sanitario de sus cuerpos-. Así, la gestión de cada vez más aspectos de sus vidas se convierte en asunto de las disciplinas biomédicas (medicina, psicología, enfermería) y de sus tecnologías y tratamientos. Este proceso, que podríamos decir que se inició con el control del parto, ha continuado en otras condiciones como el embarazo, la lactancia, la fertilidad, la menstruación, la sexualidad, la menopausia, el peso, la belleza, el tamaño de los pechos y los genitales, el malestar, la alimentación y la violencia, como ejemplos abordados en el texto.

\section{CONCLUSIONES}

En este artículo he intentado realizar una contribución en castellano que analice la biomedicalización en la vida de las mujeres, centrada en el desarrollo de este proceso en el Estado español. Para ello, se ha priorizado la producción bibliográfica local junto con algunas referencias básicas internacionales, ofreciendo una visión interdisciplinar.

Asumo que una mirada particular sobre la biomedicalización de las mujeres puede correr el riesgo de transmitir una visión esencialista: "Desde estas posiciones, el cuerpo está biológicamente determinado, es un 'alien' para los fines culturales e intelectuales, haciendo una distinción entre una mente sexualmente neutral y un cuerpo sexualmente determinado y limitado; así, lo masculino no estaría nunca limitado en su trascendencia, pero lo femenino, sí" (Esteban 2004: 32). A lo largo del artículo trato de hablar más de cuerpos, para situar las articulaciones de lo biopsicosocial, pues el cuerpo está construido y es afectado por el sistema de género patriarcal junto a la edad, la clase social, la etnicidad, la orientación sexual, la funcionalidad, renovando las similitudes y disparidades en el interior de las mujeres. Además, siguiendo a Esteban (2006), entiendo la identidad de género como algo que se hace más que es, aunque la información sanitaria solo contempla el sexo, no la orientación sexual ni otras variables relacionadas con el género. Así, Riessman (1983: 57-58) afirma que las vidas de las mujeres han sido más leídas en clave biológica y sus acontecimientos se relacionan más con los ciclos menstruales y con la menopausia; mientras que dicha interacción biológica también está en la vida de los hombres, aunque se les aplica una visión más sociocultural que aleja sus comportamientos de la presencia y acción de la testosterona. Siguiendo con esta socióloga (1983: 60), el asunto descansa en cómo ganar una comprensión de nuestra biología - yo diría de nuestra corporalidad, en general— que permita reconocer su interacción mutua con los significados y los contextos sociales. En ello será fundamental apli- 
car los sesgos de género que muestren similitudes y diferencias en la biomedicalización de los cuerpos.

Queda pendiente un análisis de las causas y consecuencias de la biomedicalización, incorporando cómo ha sido la gestión de las diferentes condiciones desde estas dimensiones, teniendo en cuenta la diversidad de cuerpos y de vidas, cómo ha sido y se ha configurado la práctica sanitaria, la construcción sociocultural de la biología y de los riesgos, junto a la agencia de los sujetos en cada contexto. Y explorando las intersecciones entre la preocupación de muchas mujeres por su salud y sus autocuidados y la percepción de que necesitan más control, pruebas y opiniones de los saberes expertos, contribuyendo a que estos (y otros) aspectos de sus vidas sigan bajo la jurisdicción de la biomedicalización, de este biopoder foucaltiano. Pero, a su vez, estas hijas de la biomedicalización están desarrollando estrategias de contestación, rompiendo con la naturalización de sus cuerpos, apostando por otras intervenciones no médicas y alejándose del control sanitario, lo que deberá ser objeto de futuras incursiones.

\section{AGRADECIMIENTOS}

A Belén Molinuevo Puras y Mari Luz Esteban Galarza, así como al conjunto de revisores, cuyas valiosas aportaciones han ampliado y enriquecido este manuscrito.

\section{BIBLIOGRAFÍA}

Annandale, E. y A. Hammarström. 2015. "A new biopolitics of Gender and Health?". Pp. 41-55 en Reimagining (Bio) medicalization, pharmaceuticals and genetics, editado por Susan E. Bell y Ann Figert. Nueva York-Londres: Routledge.

Armstrong, D. 1995. "The rise of surveillance medicine". Sociology of Health and IIIness 17(3): 393-404. https:// doi.org/10.1111/1467-9566.ep10933329.

Barceló, J. y M.J. Montes. 2016. "Cómo nacían los tarraconenses. Evolución del modelo de asistencia al parto en la Cataluña Franquista". Revista Musas 1(2): 3-19. https://doi.org/10.1344/musas2016.vol1.num2.7.

Bell, S.E. 2016. "The margins of medicalization: Diversity and context through the case of infertility". Social Science \& Medicine 156: 39-46. https://dx.doi.org/10.1016/j. socscimed.2016.03.005.

Bell, S.E. y A. Figert. 2010. "Gender and the Medicalization of Healthcare". Pp. 107-122 en Handbook of Gender and Healthcare, editado por Ellen Kuhlmann y Ellen Annandale. Basingstoke: Palgrave Macmillan.

Bell, S.E. y A. Figert. 2015. Reimagining (bio)medicalization, pharmaceuticals and genetics. Old critiques and new engagements. Nueva York-Londres: Routledge.

Bianchi, E. 2018. "Saberes, fármacos y diagnósticos. Un panorama sobre producciones recientes en torno a la farmacologización de la sociedad". Psicología, Conocimiento y Sociedad 8(2): 214-257. https://dx.doi. org/10.26864/PCS.v8.n2.11.

Bianchi, E. 2019. “¿De qué hablamos cuando hablamos de medicalización? Sobre adjetivaciones, reduccionismos y falacias del concepto en ciencias sociales". Revista Latinoamericana de Metodología de las Ciencias Sociales 9(1). https://doi.org/10.24215/18537863e052.

Biedma Velázquez, L., J.M. García de Diego y R. Serrano del Rosal. 2010. "Análisis de la no elección de la analgesia epidural durante el trabajo de parto en las mujeres andaluzas: 'la buena sufridora'”. Revista de la Sociedad Española del Dolor 17(1): 3-15. https://doi. org/10.1016/S1134-8046(10)70002-1.

Blázquez, M. 2005. "Aproximación a la Antropología de la Reproducción". Revista AIBR 42: 1-25.

Blázquez, M. 2010. Nosotras parimos ¿nosotras decidimos? La atención sanitaria al embarazo, parto y puerperio a debate. Málaga: Universidad de Málaga.

Blázquez, M. y M. Cornejo. 2014. “¿Nuevas perspectivas con respecto al riesgo? La promoción de la salud y el bienestar desde la salud holística". Pp. 4218-4238 en El encuentro entre la salud y la espiritualidad: agencia, conocimiento y prácticas, coordinado por Maribel Blázquez, Monica Cornejo y Juan Antonio Flores. Tarragona: Publicacions Universitat Rovira i Virgili.

Blázquez, M. y E. Bolaños. 2017. "Aportes a una antropología feminista de la salud: el estudio del ciclo menstrual". Salud Colectiva 13(2): 253-265. https://doi. org/10.18294/sc.2017.1204.

Bobel, C. 2009. New blood. Third wave feminism and the politics of menstruation. Londres: Rutgers University Press.

Bransen, E. 1992. "Has menstruation ever been medicalized? Or will it never happen?". Sociology of Health and IIIness 14: 98-110. https://doi.org/10.1111/1467-9566.ep11007176.

Burin, M. 1990. El malestar de las mujeres. La tranquilidad recetada. Buenos Aires: Paidós.

Busfield, J. 2017. "The concept of medicalisation reassessed". Sociology of Health \& IIIness 39(5): 759-774. https:// doi.org/10.1111/1467-9566.12538.

Camargo, K. 2013. "Medicalization, pharmaceuticalization, and health imperialism". Cadernos Saúde Pública 29(5): $\quad 844-846 . \quad$ https://doi.org/10.1590/S0102$311 \times 2013000900002$.

Camargo, R. y N. Reid. 2016. "Towards a genealogy of pharmacological practice". Medicine, Health Care and Philosophy 19: 85-94. https://doi.org/10.1007/s11019015-9648-3.

Caplan, P.J., J. McCurdy-Myers y M. Gans. 1992. "Should 'Premenstrual Syndrome' be Called a Psychiatric Abnormality?". Feminism \& Psychology 2(1): 27-44. https://doi.org/10.1177/0959353592021003.

Clarke, A., J.K. Shim, L. Mamo, J.R. Fosket y J.R. Fishman. 2003. "Biomedicalization: Technoscientific Transformations of Health, Illness, and U.S. Biomedicine". American Sociological Review 68: 161-194. https://doi. org/10.2307/1519765.

Clarke, A., L. Mamo, J.R. Fosket, J.R. Fishman y J.K. Shim. 2010. Biomedicalization. Technoscientific, Health and Illness. Durham-Londres: Duke University Press.

Colectivo de las Mujeres de Boston. 2000 [1971]. Nuestros cuerpos, nuestras vidas. Barcelona: Plaza Janés.

Colmenares, C. 2009. "La institucionalización de la maternología en España”. Historia educativa 28: 161-183.

Coll-Planas, G., E. Alfama y M. Cruells. 2013. “Se_nos gener@ mujeres. La construcción discursiva del pecho femenino en el ámbito médico". Athenea Digital 13(3): 121-135. https://doi.org/10.5565/rev/athenead/v13n3.1079.

Conrad, P. 1975. "The discovery of hyperkinesis: notes on the medicalization of deviant behavior". So- 
cial Problems 23: 12-21. https://doi.org/10.1525/ sp.1975.23.1.03a00020.

Costa, F. 2017. "Vida saludable, fitness y capital humano". Pp. 111-140 en La salud inalcanzable. Biopolítica molecular y medicalización de la vida cotidiana, editado por Flavia Costa y Pablo Rodríguez. Buenos Aires: Eudeba.

Crawford, R. 1980. "Healthism and the medicalization of everyday life". International Journal of Health Service 10(3): 365-388. https://doi.org/10.2190/3H2H-3XJN3KAY-G9NY.

De Miguel, J. 1980. "Sociología de la población y control de la natalidad en España". Reis 10: 15-47. https://doi. org/10.2307/40182773.

Delgado, A., M.C. Sánchez, I. Galindo, M.J. Duque y C. Pérez. 2001. "Actitudes de las mujeres ante la menopausia y variables predictoras". Atención Primaria 27(1): 3-11. https://doi.org/10.1016/S0212-6567(01)78765-3.

Ehrenreich, B. y D. English. 1981 [1972]. Brujas, comadronas y enfermeras. Historia de las sanadoras. Dolencias y trastornos. Política Sexual de la enfermedad. Madrid: Horas y Horas.

Ehrenreich, B. y D. English. 1990 [1973]. Por su propio bien. 150 años de consejos expertos a las mujeres. Madrid: Taurus.

Esteban, M.L. 2001. Reproducción del cuerpo femenino. Donostia: Tercera Prensa.

Esteban, M.L. 2004. Antropología del cuerpo. Barcelona: BeIlaterra.

Esteban, M.L. 2006. "El estudio de la salud y el género: las ventajas de un enfoque antropológico y feminista". Salud colectiva 2(1): 9-20. https://doi.org/10.18294/ sc.2006.52.

Fassin, D. 2011. "This is not medicalization". Pp. 85-93 en Drugs and Culture: Knowledge, Consumption and Policy, editado por Geoffrey Hunt, Maitena Milhet y Henri Bergeron. Farnham: Ashgate Publishing.

Felitti, K. 2016. "El ciclo menstrual en el siglo XXI. Entre el mercado, la ecología y el poder femenino". Sexualidad, Salud y Sociedad 22: 175-208. https://dx.doi. org/10.1590/1984-6487.sess.2016.22.08.a.

Findlay, D. 1992. "The Medical Gaze: Medical Models, Power, and Women's Health". Atlantis: Critical Studies in Gender, Culture \& Social Justice 18(1 y 2): 104-124.

Foucault, M. 1976. "La crisis de la medicina o la crisis de la antimedicina". Educación Médica y Salud 10(2): 152-169.

Fox, R. 1977. "The Medicalization and Demedicalization of American Society". Daedalus 106(1): 9-22.

Freidson, E. 1978 [1970]. La profesión médica. Barcelona: Península.

Freixas, A. 2007. Nuestra menopausia: una versión no oficial. Barcelona: Paidós lbérica.

Freixas, A. y B. Luque. 2009. "El secreto mejor guardado: la sexualidad de las mujeres mayores". Política y Sociedad 46(1): 191-203.

Gannon, L. 1998. "The Impact of Medical and Sexual Politics on Women's Health". Feminism \& Psychology 8(3): 285-302. https://doi.org/10.1177/0959353598083004.

García D. y E. Pérez Sedeño. 2017. Las "mentiras" científicas sobre las mujeres. Madrid: Los Libros de la Catarata.

García Porta, M. 2006. "Síndrome premenstrual: aproximación crítica". Revista AlBR 1(1): 80-102. https://doi. org/10.11156/aibr.010107.

Gibbon, S. 2006. "Nurturing Women and the BRCA Genes: Gender, Activism and the Paradox of Health Aware- ness". Anthropology \& Medicine 13(2): 157-171. https://doi.org/10.1080/13648470600738435.

González, J., B. Fernández y J.I. Gómez. 2015. "Historia del alivio del dolor del parto en España". Anales de la Real Academia de Medicina y Cirugía de Valladolid 52: 71-84.

González García, M. 2015. La medicalización del sexo: el viagra femenino. Madrid: Los Libros de la Catarata.

Gracia, M. 2007. "Comer bien, comer mal: la medicalización del comportamiento alimentario". Salud pública de México 49(3): 236-242. https://doi.org/10.1590/S003636342007000300009 .

Guilló, M. 2014. “Mujeres jóvenes y menstruación: contracultura y resignificación del ciclo menstrual en el País Vasco". Pp. 143-65 en Jóvenes, desigualdades y salud: Vulnerabilidad y políticas públicas, editado por Oriol Romaní y Lina Casado. Tarragona: Publicacions Universitat Rovira i Virgili.

Halfmann, D. 2012. "Recognizing Medicalization and Demedicalization: Discourses, Practices, and Identities". Health 16(2): 186-207. https://doi.org/10.1177/1363459311403947.

Hislop, J. y S. Arber. 2003. “Understanding Women's Sleep Management: Beyond Medicalization-Healthicization?". Sociology of Health \& IIIness 25(7): 815-837. https://doi.org/10.1046/j.1467-9566.2003.00371.x.

Hogle, L.F. 2005. "Enhancement Technologies and the Body". Annual Review of Anthropology 34: 695-716. https:// doi.org/10.1146/annurev.anthro.33.070203.144020.

Illich, I. 1975. Némesis médica. Barcelona: Barral Editores.

Inhorn, M. 2006. "Defining Women's Health: A Dozen Messages from More than 150 Ethnographies". Medical Anthropology Quarterly 20(3): 345-378. https://doi. org/10.1525/maq.2006.20.3.345.

Juan, M. 1991. "Crear el nacimiento: la medicalización de los conflictos en la reproducción". REIS 53: 29-51. https:// doi.org/10.2307/40183575.

Laumann, E., A. Paik y R.C. Rosen. 1999. "Sexual Dysfunction in the United States: Prevalence and Predictors". Jama 281(6): 537-544. https://doi.org/10.1001/jama.281.6.537.

Leavitt, J. 1980. "Birthing and Anesthesia: The Debate over Twilight Sleep". Signs 6(1): 147-164. https://doi. org/10.1086/493783.

Liévano, M.P. 2012. "El uso de la cirugía estética. Un acercamiento a la (re)construcción del cuerpo y la subjetividad femeninos". Tesis doctoral, Departamento de Psicología, Universitat Autónoma de Barcelona.

Lippman, A. 1991. "Prenatal Genetic Testing and Screening: Constructing Needs and Reinforcing Inequities". American Journal of Law and Medicine 17(1 y 2): 1550.

Lock, M. 1993. Encounters with Aging: Mythologies of Menopause in Japan and North America. Berkeley: University of California Press.

Lock, M. 2001. "The Tempering of Medical Anthropology: Troubling Natural Categories". Medical Anthropology Quarterly 15(4): 478-492. https://doi.org/10.1525/ maq.2001.15.4.478.

Lock, M. 2004. "Medicalization and the Naturalization of Social Control". Pp. 116-24 en Encyclopedia of Medical Anthropology, editado por Carol R. Ember y Melvin Ember. Nueva York: Kluwer Academic Plenum.

López Carrillo, M. s.f. "La medicalización de la vida y la salud de las mujeres". Consulta 19 de junio de 2019 (https:// www.caps.cat/images/stories/Medicalizacion_de_la_vida_y_la_salud_Xarxa_de_salut_Margarita_Lopez_Carrillo.pdf). 
López Carrillo, M. 2002. "Lo que deberían saber las donantes de óvulos". Mujer y Salud 9. Dossier. Consulta 19 de junio de 2019 (https://matriz.net/mys-09/dossier/doss_09_02.html).

López-Olmos, J. 2013. "Hipertrofia de labios menores y/o asimetría: petición de resección, ¿casualidad o moda? Estudio de dos periodos, 1994-2007 y 2008-2010". Clínica e Investigación en Ginecología y Obstetricia 40(1): 2-7. https://doi.org/10.1016/j.gine.2011.11.001.

Lupton, D. 1995. The Imperative of Health. Public Health and the Regulated Body. Los Ángeles: Sage.

Llácer, A. 2000. "Objetivo 8: mejorar la salud de las mujeres." Informe SESPAS 2000, la Salud Pública ante los desafíos del nuevo siglo. Consulta 5 de mayo de 2019 (https://www.sespas.es/informe2000/d1_08.pdf).

Llovet, J.J. 1991. "Las asociaciones de consumidores y usuarios y la sanidad". REIS 53: 165-195. https://doi. org/10.2307/40183580.

Martin, E. 1987. The Woman in the Body: A Cultural Analysis of Reproduction. Boston: Beacon Press.

Martínez, F., N. Parera y J. Rius. 2018. "Características e impacto de la menstruación en la mujer española: el porqué del interés de la posibilidad de supresión de la menstruación". Medicina Reproductiva y Embriología Clínica 5: 71-79. https://doi.org/10.1016/j.medre.2017.10.002.

Miglioranza, I. 2018. "Neuropolíticas del deseo y 'viagra femenino': biomedicalización, feminismo y neoliberalismo en el siglo XXI". Arxiu d'Etnografía de Catalunya 18: 95-128. https://doi.org/10.17345/aec18.95-128.

Ministerio de Sanidad y Consumo. 2007. Estrategia de Atención al Parto Normal. Madrid: Ministerio de Sanidad y Consumo. Consulta 9 de octubre de 2019 (https:// www.elpartoesnuestro.es/sites/default/files/recursos/ documents/m_sanidad_-_estrategia_de_atencion_al_ parto_normal_2007.pdf).

Montes, M.J. 1999. "Medicalización reproductiva o la alienación del cuerpo". Enfermería Clínica 5: 206-212.

Mosquera, C. 2009. "Cambios en el uso de la terapia hormonal sustitutiva tras una intervención informativa dirigida a mujeres y prescriptores". Gaceta Sanitaria 23(2): $100-$ 103. https://doi.org/10.1016/j.gaceta.2008.04.003.

Moynihan, R., I. Heath y D. Henry. 2002. "Selling sickness: the pharmaceutical industry and disease mongering". British Medical Journal 324: 886-891. https://doi. org/10.1136/bmj.324.7342.886.

Muñiz, E. 2010. Transformaciones corporales: la etnocirugía. Barcelona: Universitat Oberta de Catalunya.

Murguía, A., T. Ordorika y F. Guerrero. 2016. "Consideraciones epistemológicas en torno a la medicalización en América Latina: balances y propuestas". Ludus Vitalis XXIV (46): 99-128.

Nash, M. 2000. "Maternidad, maternología y reforma eugénica en España. 1900-1939". Pp. 628-629 en Historia de las mujeres en Occidente, editado por George Duby y Michel Perrot. Madrid: Taurus.

Nogueiras, B. 2018. "La teoría feminista aplicada al ámbito de la salud de las mujeres: discursos y prácticas (España, 1975-2013)". Tesis doctoral, Instituto Universitario de Estudios Feministas, Universidad Complutense de Madrid, Madrid.

Oinas, E. 1998. "Medicalisation by whom? Accounts of menstruation conveyed by young women and medical experts in medical advisory columns". Sociology of Health \& IIIness 20(1): 52-70. https://doi. org/10.1111/1467-9566.00080.
Ortiz, T. 1992. "Luisa Rosado, una matrona en la España ilustrada". Dynamis 12: 323-346.

Ortiz, T. 2006. Medicina, historia y género, 130 años de investigación feminista. Oviedo: KRK.

Ortiz, T. y A. Ignaciuk. 2018. "Historia de la anticoncepción e historia oral, una revisión bibliográfica". Dynamis 38(2): 293-302. https://dx.doi.org/10.4321/S021195362018000200001.

Padamsee, T. 2011. "The Pharmaceutical Corporation and the 'Good Work' of Managing Women's Bodies". Social Science \& Medicine 72(8): 1342-1350. https://doi.org/10.1016/j.socscimed.2010.10.034.

Porroche, A. 2017. "Problematizando la desinformación en las campañas de concienciación sobre el cáncer de mama". Gaceta Sanitaria 31(3): 250-252. https://doi. org/10.1016/j.gaceta.2016.11.003.

Pujal, M., P. Albertín y E. Mora. 2015. "Discursos científicos sobre el dolor cronificado sin-causa-orgánica". Política y Sociedad 52(3): 921-948. https://dx.doi.org/10.5209/ rev_POSO.2015.v52.n3.45295.

Ramos, M. 2017. Envejecer siendo mujer: dificultades, oportunidades y retos. Barcelona: Bellaterra.

Riessman, C.K. 1983. "Women and Medicalization: A New Perspective". Social Policy 14(1): 3-18.

Riska, E. 2003. "Gendering the medicalization thesis". Pp. 5987 en Advances in Gender Research, vol. 7, Gender Perspectives on Health and Medicine: Key Themes, editado por M. Texler, V. Demos y J. J. Kronenfeld. Bingley: Emerald Group Publishing Limited. https://doi. org/10.1016/S1529-2126(03)07003-6.

Riska, E. 2010. "Gender and Medicalization and Biomedicalization Theories". Pp. 147-170 en Biomedicalization: Technoscience, Health, and IIIness in the U.S., editado por Adele. E. Clarke, Laura Mamo, Jennifer R. Fosket, Jennifer R. Fishman y Janet K. Shim. Durham: Duke University Press. https://doi.org/10.1215/9780822391258-005.

Rodríguez, P. 2017. "Biotecnologías del yo". Pp. 89-110 en La salud inalcanzable. Biopolítica molecular y medicalización de la vida cotidiana, editado por Flavia Costa y Pablo Rodríguez. Buenos Aires: Eudeba.

Rodríguez Zoya, P. 2017. "El dispositivo biopolítico de medicalización alimentaria”. Pp. 141-168 en La salud inalcanzable. Biopolítica molecular y medicalización de la vida cotidiana, editado por Flavia Costa y Pablo Rodríguez. Buenos Aires: Eudeba.

Romero, C. y P. Santoro. 2018. "Hybrid Zones, Bio-objectification and Microbiota in Human Breast Milk Banking". TECNOSCIENZA 9(2): 33-60.

Romo, N. y E. Gil. 2008. "Los discursos de las mujeres en relación al consumo de psicofármacos: estrategias y causas de la medicalización". Pp. 121-126 en La experiencia de enfermar en perspectiva histórica, coordinado por Teresa Ortiz Gómez y otros. Granada: Universidad de Granada.

Rose, N. 2007. "Beyond medicalisation". The Lancet 369(9562): 700-702. https://doi.org/10.1016/S01406736(07)60319-5.

Rose, N. 2013. "The Human Sciences in a Biological Age". Theory, Culture \& Society 30(1): 3-34. https://doi. org/10.1177/0263276412456569.

Rueda, J.R. 1997. "Medicalization of menopause and public health". Journal of Psychosomatic Obstetrics and Gynecology 18(2): 175-180. https://doi. org/10.3109/01674829709085584. 
Ruiz-Cantero, M.T. y L.M. Verbrugge. 1997. "A two way view of gender bias in medicine". Journal of Epidemiology \& Community Health 51(2): 106-109. https://doi. org/10.1136/jech.51.2.106.

Ruiz-Cantero, M.T.; C. Tomás-Aznar; M.J. Rodríguez-Jaume; E. Pérez-Sedeño y Á. Gasch-Gallén. 2019. "Agenda de género en la formación en ciencias de la salud: experiencias internacionales para reducir tiempos en España". Gaceta Sanitaria 33(5): 485-490. https://doi. org/10.1016/j.gaceta.2018.03.010.

Ruiz, D. y A. Gomis. 2017. Compromiso social y género: la historia de las matronas en España en la Segunda República, la Guerra Civil y la Autarquía (1931-1955). Madrid: Ayuntamiento de Alcalá de Henares.

Sáez, C. 1988. Sobre mujer y salud mental. Barcelona: LaSal.

Sánchez, R. 2012. "Lo que una mujer no necesita". Actualización en Medicina de Familia 8(11): 617-625.

Segura, J. 2018. "Historia de la atención primaria y de la medicina social". Consulta 30 de junio de 2019 (https:// saludpublicayotrasdudas.wordpress.com/2018/04/18/ historia-de-la-atencion-primaria-y-de-la-medicina-social/).

Sfez, L. 2008. La salud perfecta. Crítica de una nueva utopía. Buenos Aires: Prometeo.

Shipman, J. 2010. "'More natural but less normal': Reconsidering medicalisation and agency through women's accounts of menstrual suppression". Social Science \& Medicine 71: 1324-1331. https://doi.org/10.1016/j. socscimed.2010.06.041.

Sholl, J. 2017. "The muddle of medicalization: pathologizing or medicalizing?". Theoretical Medicine \& Bioethics 38: 265-278. https://doi.org/10.1007/s11017-017-9414-z.

Sibilia, P. 2016. El hombre postorgánico: cuerpo, subjetividad y tecnologías digitales. Buenos Aires: Fondo de Cultura Económica.

Sweet, P.L. 2014. "'Every bone of my body:' Domestic violence and the diagnostic body". Social Science \& Medicine 122: 44-52. https://doi.org/10.1016/j. socscimed.2014.10.014.
Szasz, T. 1974 [1970]. La fabricación de la locura. Barcelona: Kairós.

Tiefer, L. 2006. "Female Sexual Dysfunction: A Case Study of Disease Mongering and Activist Resistance". PLoS Medicine 3(4): 14-18. https://doi.org/10.1371/journal. pmed.0030178.

Torres, J. 2014. "Medicalizing to demedicalize: lactation consultants and the (de)medicalization of breastfeeding". Social Science \& Medicine 100: 159-166. https://doi. org/10.1016/j.socscimed.2013.11.013.

Valls, C. 2010. Mujeres, salud y poder. Madrid: Cátedra.

Valls, C. 2018. Medio ambiente y salud. Madrid: Cátedra.

Velasco, S. 2009. Sexos, género y salud. Madrid: Minerva.

Waldby, C. y M. Cooper. 2008. "The Biopolitics of Reproduction: Post-Fordist Biotechnology and Women's Clinical Labour". Australian Feminist Studies 23(55): 57-73. https://doi.org/10.1080/08164640701816223.

Williams, S.J. 2001. "Sociological imperialism and the profession of medicine revisited: where are we now?". Sociology of Health \& IIIness 23(2): 135-158. https://doi. org/10.1111/1467-9566.00245.

Wolf, N. 1991. El mito de la belleza: cómo las imágenes de la belleza son usadas contra las mujeres. Barcelona: Emecé.

Woodward, K. 1999. "Statistical Panic". Differences. A Journal of Feminist Cultural Studies 11(2): 177-203. https://doi. org/10.1215/10407391-11-2-177.

Zafra, E. 2015. "Estudio comparativo de los procesos de socialización alimentaria de adolescentes diagnosticados y no diagnosticados de trastornos alimentarios". Revista de Humanidades 25: 103-128. https://doi.org/10.5944/ rdh.25.2015.14158.

Zola, I.K. 1972. "Medicine as an institution of social control". Sociological Review 20: 487-504. https://doi.org/10.1111/j.1467-954X.1972.tb00220.x.

Zuluaga, J.C.; J. Cruz y M. Meneses. 2018. "Medicalización, promoción de la enfermedad y disfunción sexual femenina". Revista CS 24: 41-66. https://doi.org/10.18046/ recs.i24.2308.

MARIBEL BLÁZQUEZ RODRÍGUEZ es profesora contratada doctora en la Universidad Complutense de Madrid. Doctora en Antropología por la Universidad Rovira i Virgili de Tarragona. Licenciada en esta disciplina por la Universidad Autónoma de Madrid. Primer Premio Victoria Kent (XX Edición) del Seminario de Estudios Interdisciplinarios de la Mujer de la Universidad de Málaga. Ha impartido clases en el Máster de Género y Desarrollo de la Universidad Complutense, en el Instituto de Salud Carlos III y en diferentes instituciones de salud. 